\title{
"Seahorse" Hook Plating Supplemented by a Supra- Acetabular Screw for the Fixation of Avulsion Fracture of the Ilium: A Case Report
}

\author{
Ahmad Arieff Atan*, Zamri Ab Rahman, Khairul Rizal Zayzan, Norhaslinda Bahaudin and \\ Abdul Rauf Ahmad
}

Department of Orthopaedic, Hospital Tuanku Ja'afar Seremban, Negeri Sembilan, Malaysia

*Corresponding author: Ahmad Arieff Bin Atan, Department of Orthopaedic, Hospital Tuanku Ja'afar Seremban, Jln Rasah, Bukit Rasah, 70300 Seremban, Negeri Sembilan, Malaysia

\section{ARTICLE INFO}

Received: 㠅 June 25, 2020

Published: 幽 July 06, 2020

Citation: Ahmad Arieff A, Zamri Ab R, Khairul Rizal Z, Norhaslinda B, Abdul Rauf A. "Seahorse" Hook Plating Supplemented by a Supra-Acetabular Screw for the Fixation of Avulsion Fracture of the Ilium: A Case Report. Biomed J Sci \& Tech Res 28(4)-2020. BJSTR. MS.ID.004686.

Keywords: Ilium; Avulsion Fracture; Plating; Hook; Tile A2

\section{ABSTRACT}

Isolated fracture of the iliac wing rarely causes pelvic ring instability, and therefore has favourable outcomes with non-operative management. However, among the indication of surgery includes extension of the fracture to the hip joint which causes intra-articular incongruency. A 44 years old patient presented to us with such fracture and was treated surgically with an anatomically-contoured reconstruction plate, supplemented by a supra-acetabular screw. The plate was placed on the outer table of the ilium and hooked beneath the anterior superior iliac spine (ASIS) as it wrapped the ilium until its inner table, resembling a "seahorse" on the radiograph. The biomechanical advantages of such fixation include resistance against shearing and muscular-pull forces, and good inter fragmentary compression. The patient recovered well and returned to his pre-injury activities as early as at 6 months postoperatively. Despite its diverse variants, iliac wing fractures which extend to the hip joint without significant involvement of the columns may benefit greatly from a surgical fixation using the combination of hook reconstruction plate and supra-acetabular screw fixation

\section{Introduction}

As most of the pelvic fractures are usually the result of highenergy trauma, isolated fracture involving the ilium is uncommon. The main mechanism is commonly due to direct impact and rarely involves multidirectional or rotational force displacement of the fragment. According to the widely-used Tile's classification for pelvic fractures, this injury can be classified as type A1 or A2 [1]. Although fractures involving iliac wing render the pelvic ring to be vertically- and rotationally-stable, [1] surgical intervention is indicated especially if there is any intra articular involvement. Ilium plating using a traditional ilioinguinal approach is commonly adopted as the fixation strategy. We describe an interesting case of a patient who sustained isolated avulsion fracture of the ilium and was treated surgically using a method which we believe provides a stable fixation via a simpler ilio femoral approach.

\section{Case Report}

A 44 years old male presented to us in the Casualty Department with left hip pain and inability to weight-bear immediately after a motor-vehicle accident. He was haemo dynamically stable. A detailed physical examination found that the pelvic spring test was negative but the patient was unable to flex the hip. He also had lower limb length discrepancy, with the left lower limb is shorter than the contra lateral side. No injuries to the head, chest, abdomen or other limbs were observed. The plain radiograph of the pelvis revealed the diagnosis of left iliac wing fracture with left hip sub luxation. The avulsed fragment was displaced to infero-laterally. Subsequent assessment using computed tomography (CT) scan showed simple avulsion of the iliac wing (type A2 according to Tile's classification). There is marginal involvement of the anterior column of the acetabulum. The anterior and posterior portions of the pelvic ring are intact. (Figure 1) Due to the intraarticular involvement of the fracture causing the left hip joint in congruency, surgical treatment was decided to be the best option for the patient. The patient was operated around 8 days after the trauma, while allowing the soft tissue contusion to subside. In the operating theatre, the patient was put on a supine position, under general anaesthesia. The whole 
left lower limb was allowed free and prepared together with the left hemipelvis. (Figure 2a) A limited ilio femoral approach was adopted as the approach of choice. The bony landmarks (the intact ilium and the displaced fragment) were marked, with the surgical incision planned from the posterior border of the iliac crest towards the anterior superior iliac spine (ASIS), curving distally and laterally along an imaginary line connecting the ASIS with the fibular head.

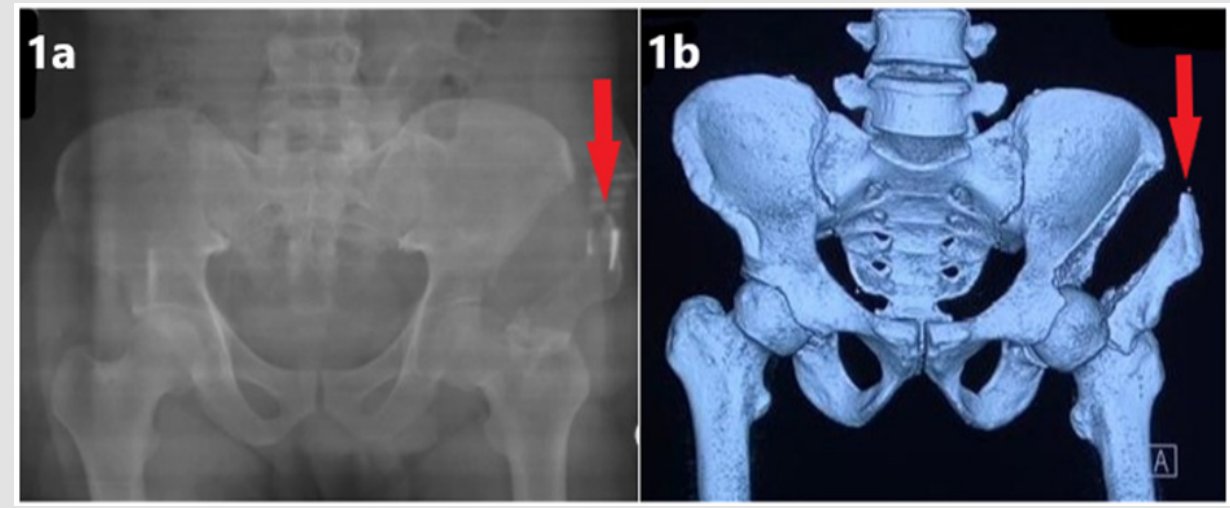

Figure 1: (1a) Antero posterior view of the pelvis radiograph showing the presence of avulsion fracture of the left iliac wing, as pointed by the red arrow.

(1b) CT scan of the pelvis confirmed the diagnosis and showed involvement of part of the articular coverage of the left hip joint. The CT scan also helped to ascertain the stability of the pelvic ring and exclude injury to other components of the pelvis.

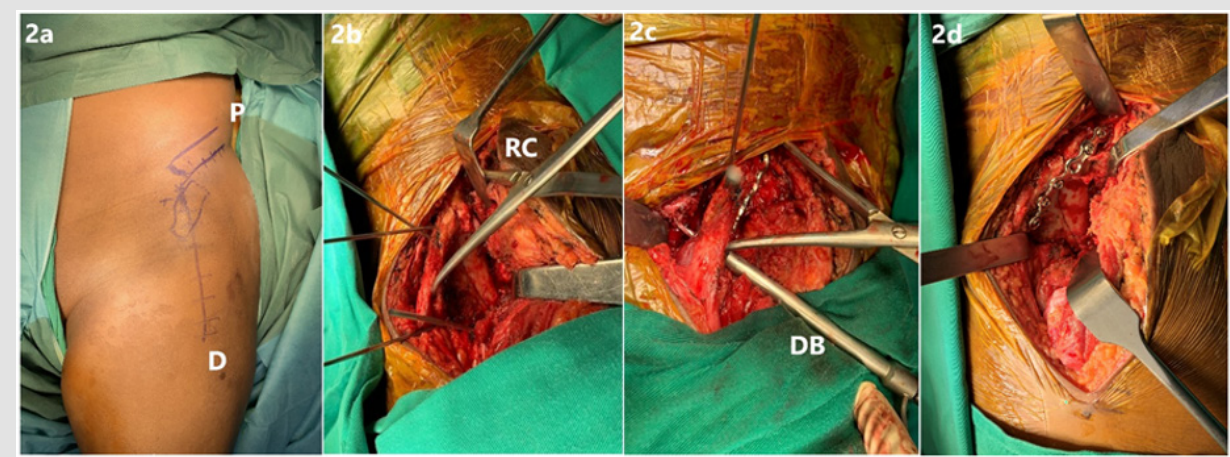

Figure 2: Intraoperative images showing the various stages of the approach, reduction and fixation of the ilium.

(2a) The bony landmarks, namely the ilium and the displaced fragment were marked, with the planned incision drawn from proximal $(\mathrm{P})$ to distal $(\mathrm{D})$.

(2b) The fracture was reduced with a reduction clamp $(\mathrm{RC})$ and temporarily held with Kirschner wires.

(2c) The drillbit (DB) penetrating through-and-through the last third hole and the last hole of the hook plate distally.

(2d) The final position of the plate on the outer table of the ilium.

Exposure of the muscular attachment on the ilium leads to subperiosteal dissection with limited elevation of the iliacus muscle on the inner table and tensor fasciae latae on the outer table of the ilium. The origin of sartorius on the ASIS was not detached. The fracture site was identified and cleared from any interposed tissue. The interval between sartorius and tensor fasciae latae was utilized to aid fracture reduction. The fracture was reduced using a reduction clamp and the reduction temporarily held with Kirschner wires. (Figure 2b). A $3.5 \mathrm{~mm}$ reconstruction plate was then used and anatomically-contoured like a 'seahorse'-shaped plate. (Figure 3) The end of the plate was contoured like a hook, and the plate was placed on the outer table of ilium, with the "hook" end wrapping around beneath the anterior superior iliac spine from the outer table to the inner table. This allowed the most distal screw to be inserted through-and-through occupying both the last and the third-last holes (Figure 2c and 4) This fixation was afterward supplemented with asupra-acetabular screw to further compressing the fragment. A 7.3mm half-threaded, cannulated screw was used, with a washer, and inserted slightly distal to the anterior inferior iliac spine (AIIS), directed towards the posterior superior iliac spine (PSIS) within the ilium column (Figure 4). There was nointraoperative and postoperative complication occurred. Post-operative radiographs showed restoration of the articular congruency of the left hip joint, with stable fixation achieved. The patient was instructed for non-weight bearing at the initial stage. After 2 months postsurgery, there was early evidence of union. Radiographic union was achieved at 3 months postoperatively and the patient was allowed to walk without support. He was pain-free returned completely to his pre-injury activities 6 months after the surgery. 


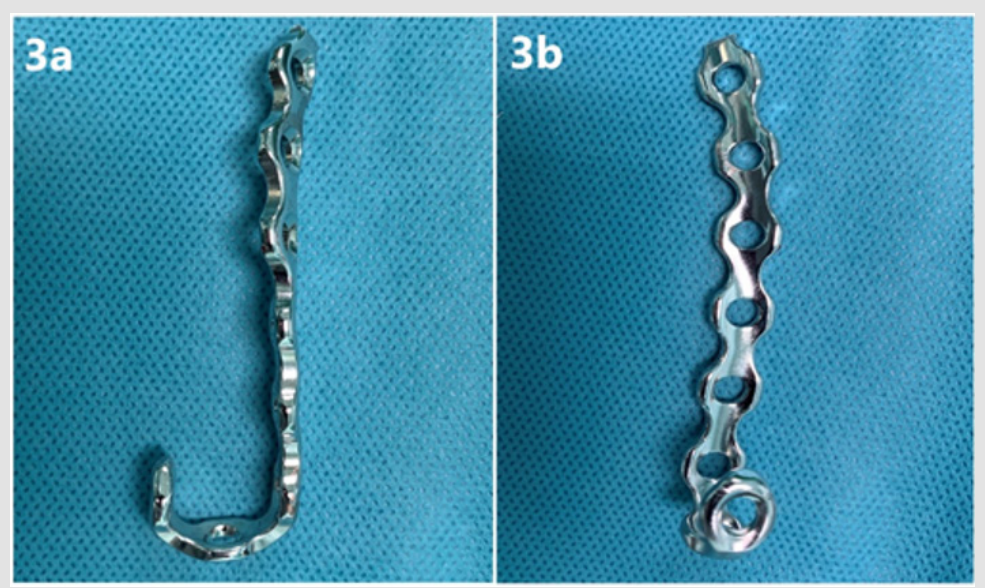

Figure 3: The "seahorse" hook plate gets its name from its appearance resembling a seahorse especially on the image-intensifier and the radiographs. The plate used was a $3.5 \mathrm{~mm}$ reconstruction plate, which was anatomically-contoured according to the shape of the outer and inner surfaces of the ilium. The distal part was shaped like a hook, to wrap around beneath the anterior superior iliac spine (ASIS).

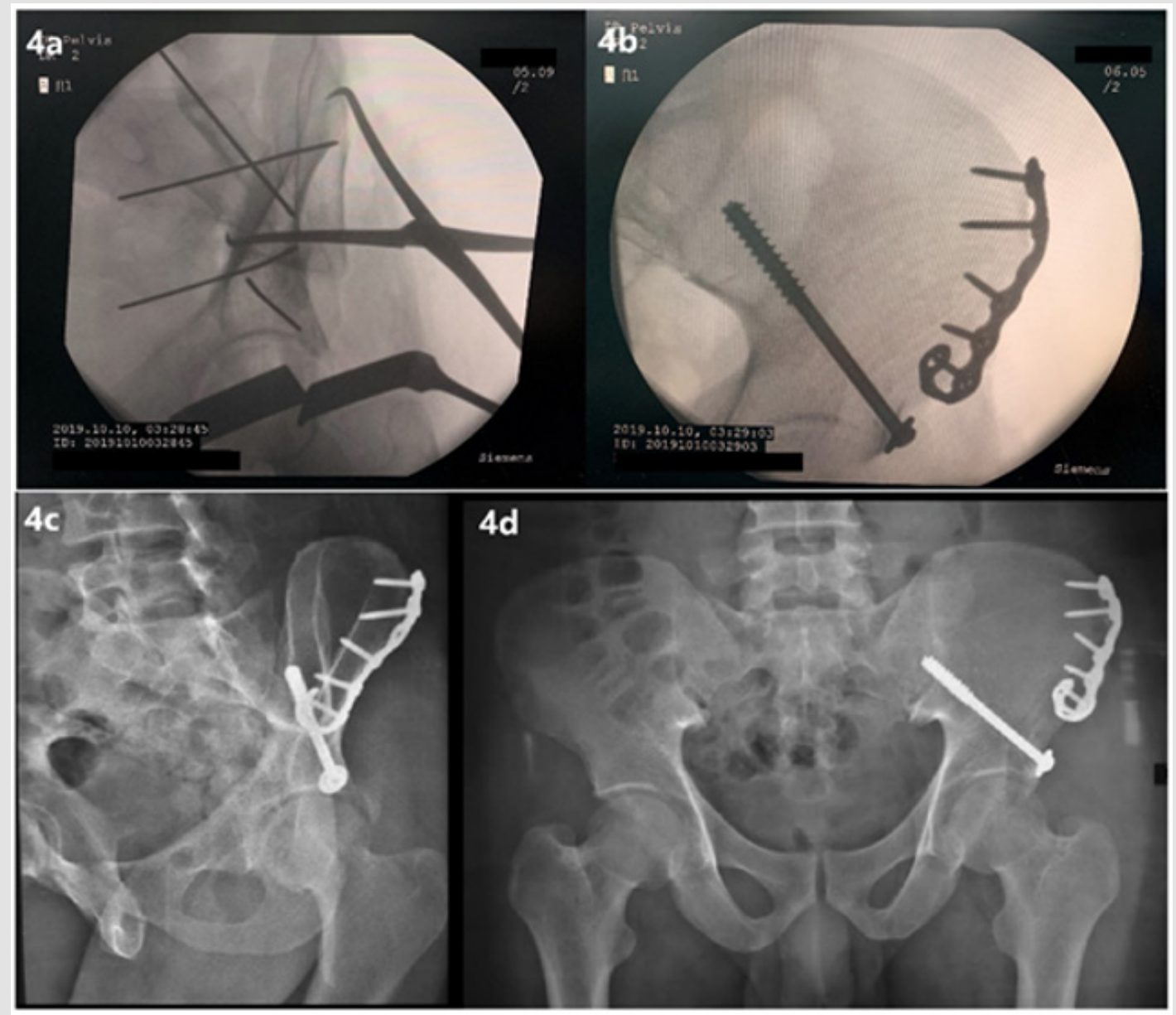

Figure 4: (4a) The picture from the image-intensifier screen, showing the anatomical reduction achieved prior to the placement of the hook plate and supra-acetabular screw. The reduction clamp and Kirschner wires used to hold the reduction are clearly visible.

(4b) The final position of the hook plate and the supra-acetabular screw was checked using the image intensifier, with the fracture site was well-reduced.

(4c \& 4d) The postoperative radiograph showing the obturator oblique and antero posterior views of the pelvis respectively-the avulsion fracture was well-reduced, with the restoration of the left hip joint congruency. The implants appeared to provide a very stable fixation for the fracture. 


\section{Discussion}

An isolated fracture of the ilium is normally considered to be stable as it does not disrupt the pelvic ring and interfere with the biomechanics of the pelvis or axial load distribution. Tile classified this fracture as type A1 or A2 and placed little significance of these fractures in terms of affecting the pelvic stability [1]. However, more studies have been carried out to include this type of fracture into the evaluation of their outcomes [2,3]. Most patients were found to have concomitant injuries but fracture stabilisation is not always mandatory [2]. Due to the likelihood of high-energy trauma causing such injury, life-threatening injuries should not be missed. Associated vascular injuries must also not be missed, with ilium fracture extending to the sciatic notch is one of the warning signs warranting an angiography [3]. In our patient, the extension of the fracture line from the iliac crest towards the acetabulum only marginally involved the anterior column of the acetabulum. Although it did not disrupt the weight-bearing portion of the hip joint, it did affect the coverage of the femoral head and congruency of the articular surface - hence, surgical intervention is indicated to overcome these two issues. Traditionally, these fractures are accessible through the ilioinguinal approach, mostly through its lateral window. The iliofemoral approach is another anterior approach which can be used especially if access towards the ilium and anterior column is needed. With many modifications and extension such as extensile iliofemoral approach, the indications for this approach has been expanded [4]. Combination with either a posterior approach (eg Kocher-Langenbeck) or a middle approach (eg Pfannenstiel) helps to gain greater access for almost all type of acetabular fractures reduction and fixation, especially the ones which involve the anterior column [4].

The limited iliofemoral approach in this case utilised minimal soft tissue dissection and the simpler intermuscular interval between the sartorius and tensor fasciae latae, void from vital neurovascular structures, to achieve optimal reduction and fixation result. It needs to be emphasised that the preservation of sartorius origin is important due to its indispensable functions in hip and knee motions. An anatomically-contoured plate - the "seahorse" plate - is postulated to provide a few additional advantages if compared to the conventional plate. Eccentrical placement of the screws near the fracture site can be achieved by both this plate and the conventional plate. However, the contoured plate hooks around the ASIS to provide better resistance against the shearing force and muscular-pull force of the sartorius. As it wraps both the inner and outer table of the ilium, it provides an opportunity for more screw placements and cortical purchases, beneficial especially for a very distal fracture.
A supplementary supra-acetabular screw is needed in this patient, as it provides better inter fragmentary compression. The big diameter and length of the screw help to gain better bony purchases. This is especially true as the screw trajectory is towards the PSIS as it covers the area of the highest bony density in the ilium -the supra-acetabular region on the lateral surface of the ilium and the area superior to the sciatic buttress [5]. This corridor, along with the iliac tubercle at the superior margin of ilium, is called iliac pillar, and has been advocated for screw fixation of the ilium [5]. Combined with the "seahorse" plate, these implants allowed a more stable fixation if compared to a single simple conventional plating alone. Despite all the advantages, the placement of this plate on the outer table and its proximity to the prominent ASIS may cause discomfort in a thin patient. More biomechanics and functional outcome studies need to be carried out before it can be ascertained to be superior to simple conventional plating alone. In conclusion, despite its diverse variants, iliac wing fractures which extend to the hip joint without the involvement of the columns may benefit greatly from a surgical fixation using the combination of hook reconstruction plate and supra-acetabular screw fixation

\section{Authors' Contributions}

Ahmad Arieff Atan conceived the original idea and acted as the manuscript's primary author. Zamri Ab Rahman and Khairul Rizal Zayzan contributed in the literature review and acted as secondary authors. Abdul Rauf Ahmad gave clinical consultation on the clinical works carried out and the writing of the manuscript. Ahmad Arieff Atan, Zamri Ab Rahman, Khairul Rizal Zayzan and Norhaslinda Bahaudin contributed in the clinical works, investigations, images and resources-gathering and followed-up the patient.

\section{Conflict of Interest and Funding Declaration}

All authors declare that no conflict of interest exists and none of the authors received any funding, directly or remotely, related to the clinical work and publication of this manuscript.

\section{References}

1. Tile M (1988) Pelvic ring fractures: should they be fixed? J Bone Joint Surg Br 70(1): 1-2.

2. Hermans E, Edwards M, Biert J (2019) Isolated iliac wing fractures: are they really that benign? Acta Orthop Belg 85(4).

3. Switzer JA, Nork SE, Routt ML Jr (2000) Comminuted fracturesof the iliac wing. J Orthop Trauma 14(4): 270-276.

4. Gupta R, Soni A (2017) Iliofemoral approach to acetabulum. Trauma Int 3(1): 14-16.

5. Kim WY, Lee SW, Kim KW, Kwon SY, Choi YH (2019) Minimally invasive surgical treatment using 'iliac pillar'screw for isolated iliac wing fractures in geriatric patients: a new challenge. Eur J Trauma Emerg Surg 45(2): 213-219. 
ISSN: 2574-1241

DOI: 10.26717/BJSTR.2020.28.004686

Ahmad Arieff Atan. Biomed J Sci \& Tech Res

(C) This work is licensed under Creative

Submission Link: https://biomedres.us/submit-manuscript.php

$\begin{array}{ll}\text { BIOMEDICAL } & \text { Assets of Publishing with us } \\ \text { RESEARCHES } & \text { - Global archiving of articles } \\ \text { - Immediate, unrestricted online access } & \text { - Rigorous Peer Review Process } \\ & \text { - Authors Retain Copyrights } \\ \end{array}$

